\title{
Arbor
}

\section{Mujer, ciencia y sociedad: las Ciencias Agrarias}

\author{
$M^{a}$ Rosario de Felipe \\ Con la colaboración de Ana Chueca, Ana $M^{a}$ Vieitez, \\ Anunciación Abadía
}

Arbor CLXXII, 679-680 (Julio-Agosto 2002), 475-499 pp.

La primera parte del artículo describe el "estatus" de la mujer en la primera mitad del siglo pasado. La mujer nacía para ser ama de casa y para ello era preparada según su situación social. Las pocas mujeres que accedían a estudios superiores no encontraban colocación o tenian que dejarla al casarse, ya que trabajar fuera del hogar, suponía un menosprecio para su esposo.

Poco a poco las mujeres fueron accediendo a la Universidad y destacando su docencia e investigación. Se citan las investigadoras del Instituto Nacional de Física y Química, alguna de las cuales cursó estudios en U.S.A. y montó en España técnicas fisicas de gran relieve.

Se citan también algunas mujeres Premio Nobel, relacionadas con la Biología, cuya mentalidad dista mucho de ser feminista, resaltando en sus comentarios la superioridad del hombre con el que compartían trabajo.

A continuación se destacan los nombres de algunas investigadoras del Area de Agricultura, primeras profesoras de investigación del CSIC, pioneras en su campo de investigación.

Por último, tanto las colaboradoras como la Coordinadora de este artículo concluyen, que no han sentido discriminación alguna con respecto a sus compañeros, a lo largo de su carrera investigadora. 


\section{Consideraciones previas}

La experiencia de las investigadoras que me han ayudado a la elaboración de este artículo y la mía propia no es proclive a concluir que exista discriminación de la mujer en el Area de Ciencias Agrarias. Sin embargo, he podido comprobar por compañeras de otras Areas del CSIC que ellas la han sufrido en una u otra etapa de su carrera investigadora.

Sin tenerse que remontar a épocas pasadas la evolución de la situación de la mujer en la investigación ha seguido, a nuestro parecer, el ritmo de su incorporación al mercado laboral. Como es sabido, todavía a comienzos del siglo pasado en los hogares españoles acomodados las hijas de familia eran aleccionadas para ser unas buenas amas de casa, saber coser y bordar, tocar el piano y en prepararse para atraer una buena dote. Muy pocas mujeres accedían a la Universidad, en contraposición a sus hermanos que optaban a ser médicos, farmacéuticos o ingenieros. Y si alguna hija de familia decidía eludir las tareas domésticas o demostraba desde el colegio dotes para el estudio, a lo mas que llegaba la familia era a pagarle la carrera de maestra. Curiosamente, por proceder mi familia del medio rural, recuerdo por comentarios de mi madre y vivencias propias que todas las maestras que iban por los pueblos de Castilla eran solteras, porque indudablemente al casarse, las mujeres pasaban a ser amas de casa y a ocuparse de la educación de los hijos, abandonando toda actividad de trabajo externo al hogar.

Las mujeres no pudieron ir a los Institutos hasta 1868 y aunque se les posibilitó ir a la Universidad no tenían preparación para ello. La primera mujer española que se matriculó en una Facultad lo hizo en el curso 1872-73 y fue en la Facultad de Medicina de Barcelona, pero es sabido que cuando esta pionera llegó a tercero, la ley le impidió seguir adelante porque su afán por hacer su carrera era una pretensión ilegal, pues con la restauración de la Monarquía (1875) se restablecieron los obstáculos culturales para la mujer.

Sin embargo, la mujer causó buena impresión en todo momento en las aulas, y así puede constatarse en un comentario de D. Gumersindo Vicuña, catedrático de la Facultad de Ciencias de Madrid, en 1875, en un Acto de apertura de curso: «Lo cierto es que hay personas del bello sexo, cuyo espíritu es susceptible de grandes progresos y no es justo cerrarles todos los caminos del saber». Estas palabras sirvieron para conceder a la mujer el acceso a las aulas, pero se les impedía el ejercicio de la profesión. La injusticia fue tal que Concepción Arenal en 1892 en el Congreso Pedagógico defendió el derecho de las mujeres a ejercer todas las profesiones, con exclusión de la de las armas. En 1890 eran 25 las españolas 
Mujer, ciencia y sociedad: las ciencias agrarias

que obtuvieron el título de licenciadas, en un momento en el que el 71,5 $\%$ de la población era analfabeta. La admisión profesional plena de las mujeres en la Universidad Española se produjo definitivamente en 1910, llegando a ser las alumnas de Enseñanza Media el $3 \%$ de todos los alumnos. Considerando únicamente las carreras de ciencias en el curso 1915/16 eran 51 las mujeres que cursaban carreras de ciencias y algo mas de 3300 varones y unos quince años mas tarde (instaurada la II República) el número de mujeres se había elevado a 414 en un total de 21.000 universitarios en toda España.

\section{Primeras mujeres investigadoras}

En 1907 se crea la Junta de Ampliación de Estudios e Investigaciones Científicas, iniciativa particularmente muy interesante en el ámbito de la formación de la investigación científica. Nació como una institución autónoma, aunque dependiente del Ministerio de Instrucción Pública, e inspirada en la ideología que caracterizaba a la Institución Libre de Enseñanza (ILE) fundada en 1876 por un grupo de profesores universitarios separados de sus cátedras y presidida por Giner de los Ríos. Para contribuir al desarrollo de la educación y la ciencia en España, aquélla Junta, que presidió D. Santiago Ramón y Cajal, utilizó preferentemente dos instrumentos: por un lado, becas para estudiar en el extranjero y por otro, crear, preferentemente en Madrid, nuevos Centros de investigación, como el Instituto Nacional de Física y Química, o ayudar a mantener algunos ya existentes, como el Museo de Ciencias Naturales, el Jardín Botánico, el Museo de antropología o el Laboratorio de Investigaciones Biológicas de Ramón y Cajal. José Castillejo fue Secretario de la Junta para Ampliación de Estudios desde su fundación en 1907 hasta 1934 contribuyendo decisivamente al éxito de la Junta como primera agencia científica española. Castillejo definió la investigación en aquél momento de este modo: la investigación es libertad, vocación, privilegio, minoría, régimen específico y flexibilidad. La Residencia de Estudiantes creada en Madrid en 1910 en la calle Fortuny fue uno de los Centros culturales y científicos auspiciados por la Junta de Ampliación de Estudios. En ella se impartían conferencias sobre temas muy variados en las que intervenían los intelectuales mas destacados del momento y en ella se alojaron Madame Curie y Einstein entre otros.

Cuando la Residencia de Estudiantes mueve su sede a su actual emplazamiento, la calle Pinar, lugar llamado entonces la Colina de los Chopos por Juan Ramón Jiménez, se crea en la calle Fortuny la Residencia 
de Señoritas en 1915. Dirigida por María de Maeztu, estuvo tutelada por el Smith College a través del intercambio de profesores de Universidades Norteamericanas y la concesión de becas para que las españolas ampliaran su formación en U.S.A. La Residencia de Señoritas sobresalió como el laboratorio donde mejor se impartían las prácticas de química y física y ciencias naturales. Al ser los laboratorios de la Facultad de Ciencias de la Universidad Central pequeños, y los presupuestos escasos, los experimentos que hacían los alumnos eran muy pocos y la Residencia de Señoritas vino a contrarrestar esta carencia. Anejo a la Residencia se montó el Laboratorio Foster, por ser $\mathrm{M}^{\mathrm{a}}$ Luisa Foster que había obtenido el doctorado en Química en la Universidad de Chicago, la encargada de organizarlo y dirigirlo. De este laboratorio salieron los mejores protocolos para impartir las prácticas de química y física, de forma que la Universidad Central concedió validez académica a sus enseñanzas. En este laboratorio aprendieron química y física las mujeres que fueron luego aceptadas para hacer investigación en el Instituto Nacional de Física y Química, dependiente de la Junta para Ampliación de Estudios e Investigaciones Científicas

La vinculación de la Residencia de Señoritas al Instituto Internacional y Federación de Mujeres Universitarias, permitió que las mujeres mejor formadas pudieran ir al extranjero, estar en centros de investigación de élite y conocer personalmente a los mejores científicos de su época. Esto autorizó a que por 8 becas concedidas a varones para completar su formación en el extranjero, una fuera para una mujer.

En 1929 la fundación Rockefeller concedió 3 millones de pesetas al laboratorio de investigaciones físicas del Instituto Nacional de Física y Química, perteneciente a la Junta para Ampliación de Estudios e Investigaciones Científicas en reconocimiento al prestigio internacional alcanzado por el laboratorio de Física, dirigido por Blas Cabrera. Este Instituto Nacional de Física y Química funcionó de forma regular hasta 1937 y contó con 36 mujeres de un total de 158 personas, lo que supone un $22 \%$ aproximadamente. Algunas de ellas habían estado ya en el extranjero completando su formación y todas habían escrito varios artículos científicos de primer autor. Dada la categoría científica de los físicos y químicos, investigadores del Instituto Nacional de Física y Química, donde sobresalieron personajes de la talla de los profesores Enrique Moles, Julio Palacios y Miguel Catalán, las 36 mujeres incluidas entre ellos, debieron ser extraordinarias. Entre ellas merece destacar las investigadoras $\mathrm{M}^{\mathrm{a}}$ Teresa Salazar, colaboradora imprescindible de Enrique Moles que tenía a su cargo las medidas de masas atómicas y otras técnicas fisicoquímicas de gran precisión, con una gran destreza, y Dorotea Barnés, quien puso 
a punto la espectroscopía Raman en el Instituto, lo que supuso un avance considerable en el conocimiento de la estructura de las moléculas, y que permaneció en la Universidad de Graz (Austria) para resolver las dificultades de montaje, publicando los resultados de sus investigaciones en los Anales de la Real Sociedad Española de Física y Química en 1932. La elección de Dorotea Barnés para hacerse cargo de introducir la Espectroscopía Raman en España estuvo basada en su gran preparación, a lo que había contribuido una beca para una estancia en el Smith College de los Estados Unidos y una beca posterior en el Departamento de Química de la Universidad de Yale. Su nombre, sin embargo, quedó en el olvido, pues por imposición de su esposo tuvo que dejar la ciencia cuando se casó,. Y como ella, otras mujeres científicas siguieron el mismo destino, pues en aquélla época los hombres no eran partidarios del trabajo de la mujer fuera del hogar.

En 1996, comentaba Dorotea Barnés a su entrevistadora: «A mi me retiró de la ciencia mi marido» e igualmente al ser preguntadia $\mathrm{M}^{\mathbf{a}}$ Antonia Zorraquino, Dra. por la Universidad de Zaragoza en tiempos de la República, si le hubiera gustado seguir trabajando después de defenđer su Tesis Doctoral, respondió: «Me hubiera encantado, pero mi marido no me dejó. En aquella época el trabajo de la mujer fuera de casa súponía un menoscabo para el hombre». Su marido fưe un eminente catedrático de Química Inorgánica.

De haber podido continuar trabajando, seguramente estas mujeres habrían sido tan famosas como lo fueron investigadores coetáneos, como Blas Cabrera, los citados Miguel Catalán, Enrique Moles, Julio Palacios y otros como Leonardo Torres Quevedo, Julio Rey Pastor, Santiago Ramón y Cajal, Arturo Duperier, etc. que han pasado a la historia de la ciencia como los mejores cerebros de aquélla época.

Desgraciadamente esta mentalidad masculina se prolongó hasta bien entrada la segunda mitad del siglo XX, y personalmente viví esta ideología en alguno de mis admiradores que me confesaron su intención de retirarme de mis estudios de Farmacia (1954) para contraer matrimonio, pues la carrera no me iba a servir para nada. La mayoría de las compañeras que terminaron conmigo y que se casaron, o bien se integraron en la farmacia familiar o no trabajaron fuera de casa, y las que sí lo hicieron, han permanecido por lo general solteras. Creo que yo soy de las pocas excepciones que he continuado ejerciendo mi profesión después de casada, fuera del ámbito familiar.

A esto se suma que la mujer hasta hace relativamente pocos años ha estado convencida de la superioridad del hombre. Por los comentarios de biólogas que han recibido el Premio Nobel puede verse que estas se pre- 
sentan, por lo general, como mujeres sencillas, faltas de ambición y poco agresivas. Por ejemplo, Bárbara McClintock, premio Nobel de Medicina 1983 , a sus 80 años, por sus investigaciones sobre los genes «saltarines» del maíz, cuando supo que le habían concedido el Premio Nobel declaró: «me parece injusto que se premie a una mujer por haber disfrutado tanto». En 1944 había sido nombrada miembro de la prestigiosa Academia Nacional de las Ciencias de los Estados Unidos y Presidente de la Sociedad de Genética aquel mismo año. Del mismo modo Rosalyn Yalow obtuvo el Premio Nobel en 1977 por sus investigaciones en el equipo del Dr. Benson, cinco años después de la muerte de éste. Su comentario fue: «Estoy absolutamente convencida de que Benson se lo merecía. Yo me acomodaba con facilidad a las personas que consideraba mejores que yo y no me importaba nada dejarle a él la preferencia, porque valía para ser el primero en todas partes».

Muchas de estas mujeres, cuando han sido preguntadas en entrevistas, muestran cierto distanciamiento de la cuestión feminista. Prefieren ser valoradas en función de su trabajo, independientemente de su sexo. En ningún momento intentan ligar sus resultados científicos y su creatividad a valores especialmente femeninos y estar rodeadas de hombres es simple casualidad.

Con respecto a la representación de la mujer en las Academias de Ciencias, la Real Academia Española de Ciencias Exactas, Físicas y Naturales admitió a la primera mujer en 1988 mientras que en Estados Unidos, la primera académica lo había sido en 1925 y en Francia Madame Curie recibió el Premio Nobel en 1903.

En los últimos 50 años, las universitarias españolas han ido poco a poco abriéndose un hueco en todas las profesiones, pero sólo un número reducido ha llegado a ocupar los puestos mas altos. Este es también el caso de la comunidad académica, pues mientras en la Universidad más del $52 \%$ de los estudiantes son mujeres, tan sólo un $10 \%$ son catedráticas, por no hablar de las que han llegado a ocupar el puesto de rectoras. Las únicas rectoras españolas han sido en España Elisa Pérez Vera de la UNED, Josefina Gómez Mendoza, de la Universidad Autónoma de Madrid y Rosario Valpuesta de la Universidad San Pablo, quien cree que esta situación se debe tan solo a una herencia, a una época pasada, en la que la mujer no tenía tantas oportunidades y además había menos mujeres estudiando. Pero está completamente segura que las mujeres serán mayoría también en la cúspide, porque las cosas están cambiando y el equilibrio hombre-mujer se va normalizando en el ámbito laboral. Poco a poco se van aproximando las posiciones, aunque todavía hay un amplio abanico de situaciones intermedias y cada caso es un caso particular. 
Mujer, ciencia y sociedad: las ciencias agrarias

\section{La mujer y la investigación en la actualidad}

La mujer en el CSIC compite con el hombre en la actualidad con relativa igualdad. No he observado a lo largo de mi carrera científica, al igual que las investigadoras colaboradoras de este artículo, agravios comparativos a la hora del acceso y de la promoción a nuevas plazas, pues el curriculum vitae es la tarjeta de identidad por la que se juzgan los méritos de cada concursante. Sin embargo, el hecho de que una vez finalizada la Tesis Doctoral el camino a recorrer por el investigador sea incierto, con un número siempre indeterminado de años entre estancias en el extranjero, y contratos más o menos duraderos, condiciona la carrera investigadora de hombres y mujeres. Trabajar en investigación seriamente implica mucha dedicación y el trabajo no se acaba cuando se abandona el centro, sino que a menudo se lleva trabajo a casa y allí donde te encuentres la cabeza sigue pensando en el experimento que no acaba de salir, en el equipamiento del que se carece, en los problemas de los becarios y contratados de tu grupo y, en mi caso, como directora del Centro de Ciencias Medioambientales, en la situación de todo el personal, ya que la dirección imprime una perspectiva diferente, más amplia de lo que constituía con anterioridad llevar el grupo o el departamento adelante.

Por otra parte, la carrera investigadora es muy competitiva, primeramente entre colegas ya que la promoción a las diferentes escalas se realiza por una selección mediante concurso de méritos, en el que es fundamental la productividad científica. En segundo lugar un científico debe competir con la comunidad internacional en sus líneas de investigación y debe ser conocido y valorado.

Para lograr este reconocimiento, es indudable que el esfuerzo que tiene que realizar la mujer investigadora es muy considerable. Se necesita una gran vocación para que no decaiga el ánimo ante un trabajo muy absorbente, aprovechar todos los minutos disponibles y sobre todo organización, pues es indudable que el ser mujer conlleva otras responsabilidades añadidas como son la educación de los hijos, y el peso de la casa. El instinto de maternidad se refleja también en la formación de los jóvenes becarios a los que se les acoge para la realización de Tesinas o Tesis Doctorales y se les acaba tomando mucho cariño, deseando para ellos lo mejor que podamos ofrecerles.

En mi caso concreto he tenido la gran suerte de que mi marido también es científico. Nos conocimos en el CSIC, haciendo nuestra Tesis Doctoral y ambos hemos dedicado con ilusión la mayor parte de nuestro tiempo a la investigación científica, sin descuidar la educación de nuestros hijos. El hecho de participar en proyectos comunes de investigación 
ha tenido para mí una gran ventaja, el poder continuar hablando en casa de nuestros problemas en el laboratorio, tratando de llevar nuestro grupo de investigación adelante. También ha tenido algún inconveniente, ya que nuestros hijos nos han llamado a menudo la atención por continuar nuestros debates científicos en casa y en su presencia.

Desde 1979 desempeño tareas de responsabilidad como jefe de Departamento, investigadora principal de proyectos, coordinadora de la reestructuración de mi Instituto, directora en funciones, directora del Centro, entre ellas. En todas estas facetas mi marido ha sido y sigue siendo mi mayor apoyo, y ha sacrificado en ocasiones su propio interés para que yo pueda lograr determinados objetivos.

Dadas estas consideraciones, es evidente que la mujer investigadora actual desarrolla en general un papel destacado, comenzando a ocupar puestos de responsabilidad, tanto en subdirecciones del CSIC (caso de la Dra. Manuela Juárez), como asesoras del Presidente (Dras. Teresa $\mathrm{M}^{\mathrm{a}}$ Mendizábal y Monserrat Torné), en la dirección de centros e institutos (30 Directoras), y como delegadas del CSIC en las Comunidades Autónomas (Dra. $\mathrm{M}^{\mathrm{a}}$ Cruz Mato), si bien seguimos en minoría en algunas escalas de gestión superiores (Comisiones de Area, Presidencia y Vicepresidencia del CSIC).

\section{La investigación científica y la vida de familia}

«Investigar, escribía Raimundo Paniker, es meterse a seguir los vestigios que algo existente, real, ha dejado a su paso». «La investigación es la búsqueda de lo que es, de la esencia de las cosas a partir de sus huellas, de sus rastros». De estas definiciones queda claro que «el saber es sólo un descubrir».

El investigador ha de estudiar, y mucho, pero «ha de hacer». Este hacer lleva a la especialización científica, no hacia la amplitud cultural. Contribución personal, modesta, callada, pero personal. Esto exige ciertas cualidades de carácter: minuciosidad, fijeza, paciencia, voluntad, calma para hacer y rehacer sin estímulos de velocidad. La inconstancia, la curiosidad sin rumbo, la efervescencia inquieta, corroen la solidez del investigador.

Pero sobre todo la investigación exige una gran dedicación, constancia y continuidad, hay que profundizar en el problema a resolver y no saltar con ligereza de unas cosas a otras dispares. Se necesita concentración, realizar un trabajo concienzudo y sin prisas pues no se trata de cubrir el expediente, de realizar las cosas "por encima" sino de terminar 
Mujer, ciencia y sociedad: las ciencias agrarias

bien el objetivo emprendido, lo cual exige orden, dedicación y perseverancia. Para lograr esto, la mujer investigadora y madre de familia necesita cerrarse a muchos estímulos, para poder seguir su ruta competentemente y cumplir bien ambos compromisos.

La continuidad es imprescindible en la investigación. No sirve de nada haber conseguido un logro científico si no perdura el esfuerzo y la continua renovación y puesta al día. Así pues el trabajo perseverante, el tesón y entusiasmo en la tarea emprendida y la dedicación exclusiva son condiciones innatas integrantes de la vocación investigadora de un buen científico. A menudo los compromisos familiares se aceptan como una carga complementaria de la tarea investigadora. Para algunas investigadoras famosas la ciencia ha sido el interés principal de sus vidas, sacrificando su vida personal y familiar.

No hay ningún motivo real para que una científica casada abandone su carrera, pero naturalmente la combinación de niños y trabajo no es del todo fácil, puesto que existe una presión emocional de responsabilidades enfrentadas. Además el trabajo en investigación requiere muchas veces una total dedicación en tiempo y en esfuerzo, difícil de compaginar con una vida familiar «equilibrada». Indudablemente el mejor marido de una científica es un científico, porque una científica casada necesita un marido comprensivo. "No es difícil para la mujer encontrar su camino en la ciencia y ser esposa y madre, a condición de que elija la pareja adecuada para compartir su vida». La autora de esta afirmación Rita Levi Montalcini, ha vivido siempre soltera en el domicilio de su hermana gemela. Es evidente que el compromiso intenso personal con la ciencia y la responsabilidad familiar paralela no son siempre fáciles de combinar.

Sin embargo, investigar es, para algunos y algunas, haber aprendido unas técnicas y pasarse la vida aplicándolas, degenerando su trabajo en rutina. Nada más lejos de la vocación investigadora que convertir la investigación en rutina. El investigador ha de tener afán de superación. Los jóvenes investigadores necesitan estímulo, orientación, dirección y ejemplo de laboriosidad y solidez por parte de sus formadores. La elección de un buen maestro es indudable que influirá muy positivamente en la carrera científica de los jóvenes que se inician y es notorio, salvo casos excepcionales, que las investigadoras del CSIC tomamos en esta tarea gran responsabilidad, pues es difícil separar nuestra vocación científica del instinto maternal. Este aspecto es especialmente patente en las investigadoras solteras que han sabido crear un ambiente muy acogedor y familiar en sus laboratorios, creando vínculos de verdadera amistad con el personal joven de su grupo de investigación. 
Antes, la investigación aislada, espontánea, personal, era el ideal o «chifladura» de una vida. Hoy, la investigación profesionalizada ha de ser medio de vida. Pero que este medio de vida no extinga nuestro ideal. Muchas mujeres del CSIC conservamos nuestra vocación inicial a pesar de los muchos esfuerzos y sacrificios que nos ha costado llegar al estado actual, luchando por consolidar nuestros grupos de investigación y dotar nuestros laboratorios del equipamiento necesario para trabajar.

Esperemos que las generaciones futuras lo tengan mas fácil y que en estas tareas la mujer casada y madre de familia tenga más facilidades por parte de los estamentos gubernamentales para poder atender su investigación y su casa. La sociedad debe asumir como propio y necesario el conocimiento de la ciencia y sus aplicaciones prácticas, las nuevas tecnologías que son auténticos motores de la economía y la industria. Conseguir esto requiere el apoyo de nuestras instituciones, especialmente de los poderes públicos, cuyo compromiso con la investigación y el desarrollo científico debe ser permanente. Esperemos también que la iniciativa privada sea mas participativa en un futuro próximo, pues si bien algunos empresarios han comprendido la rentabilidad de las inversiones en investigación, aún nos falta mucho para alcanzar los niveles que dedican a este fin los principales países de nuestro entorno.

Finalmente, veo el futuro con respecto a la asunción de la mujer investigadora en este tipo de responsabilidades con gran optimismo, en las diferentes facetas del desarrollo científico. Creo positivamente que el siglo XXI es el de las mujeres y el CSIC no va a quedarse al margen de este cambio. Confío que si hoy son treinta las mujeres Directoras de Centros e Institutos del CSIC, de las cuales tres pertenecen al área de Ciencias Agrarias, este número se va a ver incrementado rápidamente en un futuro muy próximo.

\section{LA MUJER EN EL ÁREA DE CIENCIAS AGRARIAS}

El área de Ciencias Agrarias es una de las más multidisciplinares del CSIC, que se ha ido enriqueciendo con la evolución paulatina de los conocimientos científicos en materia agrícola y ganadera, de tal modo que ha surgido recientemente una subárea denominada: «Fisiología, Bioquímica y Biología Molecular de plantas» hacia donde han derivado un gran número de investigadores.

El área de Ciencias Agrarias tiene su origen en los Centros de Edafología que el CSIC fue creando en diferentes provincias españolas a partir de 1942, cuando se creó el primer Instituto de Edafología, Ecología y 
Fisiología Vegetal en Madrid, por D. José María Albareda, primer Secretario General del CSIC. Todos estos Institutos que constituyeron el Instituto Nacional de Agrobiología, encuadrado en el Patronato Alonso de Herrera, centraron sus objetivos en la resolución de los problemas agrarios de cada región, extendiendo su colaboración a Diputaciones, Cajas de Ahorros, Cajas Rurales, y al sector empresarial. De este modo los Institutos recibían ayudas económicas externas a la vez que los investigadores conocían de cerca los problemas locales agrarios y trataban de subsanarlos. Así pues, estos centros nacieron con una vocación claramente agraria, formados por miembros dedicados al estudio del suelo, en cuanto a sus propiedades físico-químicas, para su caracterización e inclusión en la clasificación general, al estudio de los componentes minerales del suelo, materia orgánica, etc., siendo destacables los trabajos sobre cartografía de suelos realizados en el Instituto de Edafología de Madrid donde se elaboraron los primeros mapas de suelos. Otros investigadores de estos Institutos centraban su atención mayormente en cultivos de interés agronómico, en cuanto a la nutrición mineral, nutrientes esenciales, fertilización de suelos y enfermedades de las plantas.

En los Departamentos de Fertilidad de Suelos se realizaban trabajos de ayuda a los agricultores con el fin de aumentar la producción vegetal, que consistían esencialmente en el diagnóstico nutricional del suelo sobre estados carenciales, vocación de los suelos para determinados cultivos, etc. También se realizaron ensayos en los invernaderos sobre nutrición de plantas, tomate, maíz, trigo, judía, remolacha azucarera; estudios de translocación de elementos, problemas de salinidad, competitividad entre nutrientes. Varias mujeres, profesoras de investigación, destacaron por su dedicación a la investigación, en el Instituto de Edafología de Madrid, la mayoría solteras y que mantuvieron su vocación investigadora hasta la jubilación. Entre ellas, estaba la Dra. Narcisa Martín Retortillo, profesora de Instituto que realizó trabajos pioneros sobre las materias húmicas del suelo con el Profesor José María Albareda cuando el Instituto de Edafología de Madrid se iniciaba como un Laboratorio de Química del Suelo, en el Edificio Rockefeller. Su tesis doctoral versó sobre análisis de turberas en España.

La Profesora Josefina Pérez Mateos, pionera en la composición mineralógica de los constituyentes del suelo, sobre minerales del suelo, nació en 1905 en Ciudad Rodrigo (Salamanca). Estudió en el Instituto Cardenal Cisneros de Madrid donde conoció a D. Celio Arévalo, uno de sus profesores que influyó mucho en su vocación hacia las Ciencias Naturales. Llevada por esta vocación inició sus estudios superiores en la Facultad de Farmacia, en la calle de la Farmacia de Madrid, siendo un pequeño 
número de mujeres las que estudiaron en esa época, que tenían reservado el primer banco de la Facultad para ellas. En 1944 leyó su Tesis Doctoral para obtener el grado de Doctor en Ciencias Naturales recibiendo el Premio Extraordinario de Doctorado por la Universidad de Madrid. En 1946 ingresó en el CSIC y obtuvo una plaza de Colaborador Científico por oposición. Realizó investigación en la Escuela Oficial de Agricultura en Wageningen (Holanda), siendo a su regreso nombrada Investigador Científico del CSIC por oposición. Creó a continuación la Sección de Petrografía Sedimentaria, posteriormente llamada Sección de Mineralogía de Suelos en el Instituto de Edafología, Ecología y Fisiología Vegetal, de la que fue Jefe hasta su jubilación en 1975.

En 1970 Josefina Pérez Mateos fue nombrada Profesor de Investigación. Como Jefe de la Sección de Petrografía Sedimentaria fue Miembro de la Junta de Gobierno del Instituto de Edafología, la única mujer miembro de la Junta. Durante el amplio periodo de su jefatura, se dedicó plenamente a la investigación. Fue una de las primeras mujeres científicas del CSIC. Supo desarrollar un ambiente científico acogedor y dejó escuela. Entre las investigadoras actuales fieles a esa escuela son de mencionar las Dras. Ascensión Pinilla y Trinidad Aleixandre, verdaderas especialistas de mineralogía de suelos.

Otra profesora de investigación, Pilar Sánchez Conde, también tuvo una actuación muy señalada en la década de 1950 poniendo en marcha nuevas técnicas en nutrición vegetal, como el conocimiento del diagnóstico nutricional por el análisis foliar, técnica de gran impacto en las décadas posteriores para hacer un seguimiento del estado nutritivo de las plantas. Son de destacar sus experimentos, realizados en invernadero, de cultivos hidropónicos.

La profesora María Dolores Angulo destacó por sus trabajos de Citogenética creando un laboratorio de referencia sobre estudios en Drosophila. El personal de este laboratorio estaba formado mayoritariamente por mujeres ayudantes diplomadas de investigación de la Escuela de Auxiliares del CSIC, que tuvo su sede en la calle Jorge Manrique. Todas ellas eran personas muy capacitadas, especializadas en diferentes técnicas que facilitaron el desarrollo de esta línea de investigación en el CSIC, donde se formaron destacados investigadores de la talla de los Prof. Giménez Martín, Antonio García Bellido, entre otros, manteniendo intercambios con el Prof. Cámara de Portugal, eminente figura en el campo de la Citogenética.

Desde su creación, los Institutos de Edafología colaboraron con las Diputaciones en problemas prácticos, prestando asesoría técnica a los agricultores de cada región, desplazándose a la zona para tomar las muestras 
o analizando los suelos que ellos traían a los Institutos distribuidos por distintas regiones de España. La recogida de las muestras se realizaba normalmente por personal investigador. Por lo general las mujeres no salían a muestrear en el campo, no sólo en el caso de trabajos rutinarios, sino tampoco incluso para sus investigaciones científicas propias, por considerarse tareas duras para ser realizadas por una mujer.

Se llevaron a cabo Planes de Desarrollo en Madrid y en Zaragoza para el estudio de la remolacha azucarera en las zonas remolacheras del Valle del Duero en Palencia, Valladolid, León y Ávila y en la zona agrícola de la cuenca del Ebro. Hubo planes también para el estudio de los cítricos en Murcia y en Valencia, en los cuales tuvo un gran protagonismo el Departamento de Fertilidad de Suelos y Nutrición Vegetal y el de Fitopatología del Instituto de Edafología y Biología Vegetal de Madrid, enviando a sus técnicos en campañas de varias semanas. En estas campañas nunca participaban mujeres, aún cuando luego intervenían en los análisis de laboratorio

Todos estos trabajos estaban orientados a la ayuda a los agricultores para mejorar la producción y la calidad de los cultivos, mejorar la fertilidad de los suelos y la nutrición mineral de las plantas. Se recomendaba el cultivo de los productos agrarios de acuerdo a las características de la zona: cerealista, vinícola, olivarera, frutera, etc., atendiendo a los parámetros mencionados.

En 1974 se reúnen en la isla de La Toja un grupo de científicos y profesores universitarios para crear la Sociedad Española de Fisiología Vegetal con el fin de coordinar los trabajos que se hacían sobre los mecanismos fisiológicos de las plantas, su metabolismo, estreses bióticos y abióticos, etc. Asisten a esta reunión cinco mujeres: la Dra. $\mathrm{M}^{\mathrm{a}} \mathrm{Cruz}$ Mato, actual Delegada del CSIC en Galicia, y las profesoras $\mathrm{M}^{\mathrm{a}}$ Teresa Piñol, Charlot Postchreiner, Jone Aguirreolea, y Adelina Vázquez, del Centro de Investigaciones Agrobiológicas de Galicia. Esta Sociedad tiene en la actualidad 583 socios y de ellos 269 son mujeres, lo que corresponde a un 46\%. Anterior a la creación de la SEFV fue la Sociedad Española de Bioquímica, integrada por bioquímica animal y vegetal, donde hay un grupo numeroso de bioquímicas españolas.

En 1976 la Asociación de Personal Investigador del CSIC (API) organizó unas Jornadas Internacionales sobre «La investigación científica y el problema agrario» que fueron coordinadas por el Prof. Valentín Hernando, Presidente del Patronato Alonso de Herrera, y por el Presidente de la API. Las Jornadas tuvieron como principal objetivo la puesta al día de la investigación agrícola en España y en diferentes países del mundo para tener una visión de conjunto y ser también conscientes de la crisis 
agraria planteada. Se requería un cambio en la agricultura, en sus diferentes aspectos científicos, tecnológicos, sociales y económicos, poniéndose de manifiesto un enorme interés del personal investigador por conocer la realidad del problema agrario y tratar de resolverlo. La Prof. Teresa Mendizábal, secretaria de la Junta Directiva de la Asociación de Personal Investigador y actual Vocal Asesora del Presidente del CSIC, tuvo un protagonismo especial en la organización de las Jornadas. El programa abarcó desde el tema general de la agricultura en el mundo, pasando por las técnicas de mecanización, fertilización, y producción animal hasta temas específicos de España, entre los que destacan el regadío, el secano y la ganadería por su importancia en el desarrollo del país. En estas jornadas intervino el Dr. Aurelio Peccei, Presidente del Club de Roma, que mostró su preocupación por la economía mundial y por las técnicas agrarias empleadas en los países desarrollados y no desarrollados y señaló los problemas energéticos que se iban a plantear por el uso masivo de los fertilizantes.

Algunos científicos de Nutrición Vegetal fueron orientando sus objetivos hacia investigaciones mas básicas (fisiología y bioquímica vegetal) y creando nuevos Departamentos en los Institutos de Edafología del CSIC. Así ocurrió en el Instituto de Edafología y Biología Vegetal de Madrid con la creación de la Unidad Estructural de Investigación de Biología Vegetal, de la que fui su primer Jefe, entre 1979 y 1990, con un grupo de investigadores procedentes del Departamento de Fertilidad de Suelos y Nutrición Vegetal del Instituto, entre los que se encontraba la Dra. Pilar Golvano, que continúa en este Departamento.

Estas Unidades de investigación o Departamentos de investigación básica se crearon en otros instituto del área de Ciencias Agrarias, como en la Estación Experimental del Zaidín (Granada) donde existe un Departamento de Bioquímica muy competitivo, en el que hay que mencionar a las doctoras Ana Chueca y $\mathrm{M}^{\mathrm{a}}$ Isabel Rodríguez, expertas en Bioquímica y Biología Celular de plantas respectivamente, así como las doctoras Rosario y Concha Azcón del Departamento de Microbiología del Suelo y Sistemas Simbióticos. La necesidad de la aplicación de las técnicas moleculares ha enriquecido estos Departamentos con jóvenes científicos expertos en biología molecular. Igualmente ha ocurrido en el Centro de Edafología y Biología Aplicada del Segura en Murcia, donde la Dra. Francisca Sevilla realiza estudios básicos de nutrición vegetal con ayuda de técnicas bioquímicas y moleculares. La Estación Experimental de «Aula Dei» (Zaragoza) ha contado entre sus especialistas en ciencias agrarias con una investigadora recientemente jubilada, Concepción Tabuenca, dedicada al estudio de nutrición de frutales, en el Departamen- 
to de Pomología, y actualmente con las Dras. Monona Abadía y María Herrero, destacadas especialistas en fisiología vegetal y biología celular de plantas, respectivamente. En Aula Dei, de los 27 investigadores de plantilla, diez son mujeres, un 37 por ciento.

En el Instituto de Investigaciones Agrobiológicas de Galicia, han desarrollado su carrera investigadora las Dras. María Cruz Mato y Adelina Vázquez, expertas en fisiología vegetal, y la Prof. Tarsi Carballas, en biología del suelo. La Prof. Ana $\mathrm{M}^{\mathrm{a}}$ Vieitiez, pionera junto a su esposo, el prof. Ballester en estudios de cultivo in vitro representa un importante puntal femenino para el futuro de este Instituto.

La Dra. Mendizábal fue, entre 1989 y 1993, coordinadora del área de Ciencias Agrarias del CSIC. Su actividad fue muy intensa en ese período, realizando un gran número de visitas a los Centros e Institutos del Área, introduciendo innovaciones científico-tecnológicas e impulsando el desarrollo de las ciencias agrarias en razón a las tendencias europeas existentes.

El aumento de la producción agraria en estos últimos cincuenta años ha convertido la agricultura en una actividad intensiva, que causará el agotamiento de los combustibles fósiles y ocasionará la contaminación de los suelos por el uso masivo de fertilizantes y fitosanitarios. Por ello la comunidad científica actualmente se inclina por una agricultura mas respetuosa con el medio ambiente, que permita la continuidad de las técnicas agrarias de forma sostenible. Todo ello conduce a una Nueva Agricultura, a una agricultura sostenible, con capacidad para mantenerse o prolongarse en la agricultura del futuro. El desarrollo sostenible, en general, es aquél que satisface las necesidades del presente, sin comprometer la capacidad de las futuras generaciones para satisfacer las propias: "ver el mundo en su conjunto». Esto supone un cambio de mentalidad en toda la sociedad, pues podemos llegar a puntos de no retorno. Este cambio de las prácticas agrícolas lleva a la puesta en marcha de biotecnologías limpias en agricultura y dentro de ellas el uso de biofertilizantes y de biocontrol de plagas y enfermedades de las plantas.

Pionera del uso de biofertilizantes fue la Prof. Eulalia Cabezas de $\mathrm{He}-$ rrera, verdadera experta en el CSIC de los estudios sobre fijación biológica de nitrógeno en el Instituto Jaime Ferrán de Microbiología. En este tema comenzamos una colaboración en 1982 ya que mi grupo de investigación venía trabajando en fijación de nitrógeno por leguminosas de grano, veza y altramuz. Al producirse la jubilación de la Dra. Herrera (1984), la Dra. Mercedes Fernández-Pascual, que se había formado con ella, se incorporó a nuestro grupo potenciando así las líneas del Departamento de Fisiología y Bioquímica Vegetal, especialmente en técnicas 
de microbiología. Nuestro Departamento se compone en la actualidad de un total de 16 personas de las que 11 son mujeres.

$\mathrm{El}$ número total de mujeres en mi Instituto que componen los diferentes Departamentos y Servicios asciende a 108 frente a un total de 203 personas, lo que corresponde a un 53 por ciento de mujeres.

Finalmente, la distribución de las mujeres investigadoras por escalas dentro del Area de Ciencias Agrarias es la siguiente:

\begin{tabular}{|l|c|c|c|c|}
\hline \multicolumn{1}{|c|}{ ESCALAS } & Hombres & Mujeres & Total & \% mujeres \\
\hline Profesores de investigación & 29 & 7 & 36 & 19 \\
Investigadores Científicos & 37 & 20 & 57 & 35 \\
Científicos titulares & 83 & 63 & 146 & 44 \\
TOTAL & 149 & 90 & 239 & 38 \\
\hline
\end{tabular}

\section{Bibliografía}

"Un siglo de ciencia en España». 1998-1999. José Manuel Sánchez Ron. Residencia de Estudiantes.

"Consideraciones sobre la investigación científica». 1951. José María Albareda. C.S.I.C.

\section{Testimonios de las científicas del área}

A continuación se adjunta el testimonio de las investigadoras que han colaborado en este trabajo.

\section{Ana Chueca. Estación Experimental del Zaidín (Granada)}

Al terminar mis estudios de Licenciatura de Farmacia quise seguir ampliando conocimientos de forma ya mas selectiva y, dado que Bioquímica había sido una de las materias que mas me impactó, decidí hablar con quien me había impartido la asignatura, el Prof. D. Ángel Santos Ruiz. Muy animada después de recibir su venia, me encontré haciendo mi Tesis Doctoral en el Laboratorio de Bioquímica de la Facultad de Farmacia de la Universidad Complutense de Madrid bajo su dirección y la de la Dra. Carmen García del Amo.

Desde entonces, junto a otros Licenciados como yo, formé parte como Profesor Ayudante de la Universidad de Madrid, al mismo tiempo que 
Mujer, ciencia y sociedad: las ciencias agrarias

becaria de Iniciación a la Investigación del Instituto de Fisiología y Bioquímica, un centro mixto con personal y apoyos de la Universidad de Madrid y del Consejo Superior de Investigaciones Científicas. El ambiente era espléndido, trabajé muy a gusto, pues reinaba una armonía que hacia posible que cada uno de los cinco grupos en que estaba estructurado el centro llevase a cabo la investigación independiente que se habían fijado. Cada grupo contaba con personal científico fijo de ambas Instituciones, amén de una pléyade de becarios con sus problemas científicos y aficiones particulares que nos unían en nuestros ratos de descanso y ocio. Aproximadamente la representación masculina y femenina era del 50 por ciento, aunque podía variar según los años. Casi todos terminamos la Tesis, y fue entonces cuando nos separamos al empezar una nueva vida profesional, dirigiéndonos a la Universidad o al CSIC y los más a la Industria Farmacéutica, según nuestras aptitudes y preferencias. Por lo que a mi respecta, una vez leída mi Tesis disfruté de una beca posdoctoral del MEC para trabajar en el Institute of Cancer Research, Department of Biophysics en Sutton (Gran Bretaña) durante el año 1967. Al volver, quede adscrita ese mismo año al Instituto de Fisiología y Bioquímica de Madrid como Ayudante Científico del CSIC, organismo al que desde entonces pertenezco.

Desde mi experiencia personal, el acceso a la carrera científica ha cambiado bastante en la actualidad. La competencia es mucho mayor, y eso hace que la edad de los candidatos se haya elevado unos cuantos años con el consiguiente desgaste por parte de los jóvenes. Si nos remontamos al nivel de becarios, prima el expediente académico. Se busca el 3, media de sobresaliente, como mínimo, y en ello las mujeres suelen dar la talla; con harta frecuencia se inclina la balanza hacia ellas en la obtención de las becas, por lo que actualmente y con justicia es frecuente ver por los laboratorios muchas chicas llenas de entusiasmo, responsables, que trabajan muy competentemente codo a codo, con los chicos. En mi Centro en la actualidad las mujeres becarias son el 62\%, mientras que el de seniors funcionarias representa el $46 \%$. En resumen, el aumento de las mujeres que dedican sus esfuerzos a la Ciencia es espectacular. Pienso que la situación será parecida en todo el área de Ciencias Agrarias, y me atrevería a decir lo mismo en cualquiera otra del CSIC. Este aumento tan considerable predice un futuro venturoso de la mujer en el CSIC y en general, en la ciencia.

El acceso a la carrera investigadora es igual para todo el CSIC, sea cual sea el Área. Por supuesto se necesita ser Doctor, y un bagaje mas que regular, que va en aumento, de becas, publicaciones, y años de estancias en laboratorios extranjeros. Esto último se considera primordial, 
constituye una criba por su dureza, y nos forma científica y humanamente para la vida científica futura. A mi entender, ya se perciben anomalías en el momento de adjudicar plazas según temas. A veces salen plazas casi con nombre y apellido, y otras derivan en una batalla campal para ver donde se ubican y quien o que grupo se queda con la misma, decidiéndose definitivamente con el nombramiento de tribunales. La designación a dedo de sus miembros, así como la falta de política científica hace que no siempre se integren los mejores en las filas del CSIC, ni sean los más fructíferos para la Institución. El problema es casi el mismo en los concursos de promoción, algo escasos de plazas en la actualidad. Hay muy buenos currículos para pocas plazas, lo que se agrava aún más con la designación a dedo de los tribunales que deciden.

A lo largo de los años, mi situación personal puedo decir que casi nunca ha estado marcada por mi condición de mujer. Tanto en mi acceso al organismo como en general en todos los concursos y oposiciones en que he participado, el resultado ha estado mas bien dirigido por las apetencias de los diferentes tribunales o de alguno de sus miembros, lo que como he dicho, ocurre también ahora. Según mi parecer y salvo las excepciones consabidas a este nivel el método de elección no intenta captar científicos según sexo, sino hacerse con los mejores especialistas según currículo y méritos científicos. Por ello, creo que la proporción de mujeres y hombres en el Organismo se debe solo a circunstancias externas, siendo el aumento en el número de mujeres paralelo a la progresiva presencia de la misma en la Universidad.

En cuanto a la ocupación de puestos directivos en el CSIC por la mujer, cabe distinguir varios factores. En principio, cabe pensar está relacionado con la, ya mencionada, escasa presencia de la mujer en la Universidad durante un tiempo, aunque en la actualidad pienso que su ausencia en puestos directivos pueda ser debida a la inercia propia de cualquier proceso aun no totalmente desarrollado, como es la presencia de la mujer en la investigación. Otra causa se puede relacionar con una falta de confianza en nosotras mismas para presentar batalla cuando se trata de ocupar algún puesto directivo. Por otra parte, la experiencia demuestra que cualquier elección esconde siempre la salvaguarda de una política propia o la conservación del sillón, lo que induce a elegir personal apropiado, o a eliminar al que suponen causará algún problema. Y aquí sí quiero, y me parece puedo, inculpar al elemento masculino del CSIC. Pensando bien, digamos que por pura costumbre de ver siempre en el sillón a un hombre no se han percatado que compañeras preparadas podrían haber hecho lo mismo, igual o mejor. Pensando algo peor, indudablemente ha habido y hay personas ocupando puestos directivos en la 
Mujer, ciencia y sociedad: las ciencias agrarias

Institución con sus ribetes machistas, o que harto tienen con la competencia de sus congéneres, como para abrir las puertas a las mujeres. Salvar su sillón les da suficiente trabajo y, además, según ellos la mujer ya manda suficiente en casa.

De ahora en adelante espero que las mujeres, que estamos ya plantando batalla, demostrando la valía en otros niveles, no nos amilanemos y, como otras muchas en otros campos, sepamos responsabilizarnos y llegar a ocupar esos puestos por derecho propio, sin necesitar cupos del 25 ni del 50 por ciento. Por mi parte, que fui elegida jefa de Departamento con anterioridad, sufrí mi acoso particular en la elección a Director a Centro. No obstante, tengo fe en las mujeres, que son las que con su trabajo y tesón conseguirán ganar ese pulso con sus compañeros los hombres. También apunto que otros problemas aflorarán y debemos estar preparadas para cuando, al competir todos, como ahora ocurre entre ellos, no siempre los mejores consiguen el puesto. Sigo dando ánimos a las féminas para prepararse a ocupar puestos directivos, y acceder a ellos a la par que nuestros colegas hombres.

Otro tema a tocar relacionado con la carrera investigadora de las mujeres son los de la familia, en especial los hijos, la casa, y los familiares mayores, problemas que hoy en día también afectan a los maridos y compañeros sentimentales. Tan asumido está este problema que ambos pueden alternativamente pedir y disfrutar los permisos por hijos. Los niños requieren dedicarles mucho tiempo, cariño y cuidados, pero eso tanto puede dárselo el padre como la madre. Mi pensamiento sobre estos problemas, cuando los tuve y también ahora, es que es necesario elegir bien, y contratar a alguien que pueda suplir a los padres en los tiempos de trabajo, sin desdeñar por otra parte sacrificar algún momento, e incluso llevar a un ritmo menos acelerado que de costumbre, la investigación en curso, durante un tiempo determinado. Recuerdo que tuve un concursooposición a Investigador Científico cinco días después del nacimiento de mi segundo hijo, y mi marido hizo las veces de padre y madre durante esos días. Todo fue bien, aunque tengo que decir que aun haciendo una buena oposición, no la gané, y no fue por este motivo, el tribunal estaba ya decantadísimo, y yo no tenia a nadie de mi parte. En conclusión, no soy partidaria de abandonar totalmente nuestro trabajo durante el crecimiento de los hijos. Normalmente se desfasa una tanto que quizás la vuelta sea ya irreversible.

Según mi idea de que la ciencia progresa por personas que nos diferenciamos sólo por circunstancias accidentales, no por ser de uno u otro sexo, no tengo mucha confianza de que las mujeres marquemos muchas diferencias respecto a lo que actualmente se está llevando a cabo por to- 
dos. Antes bien, el progreso vendrá dado por hombres o mujeres con visión de futuro e ideas claras, capaces de ponerlas en práctica, por propia evolución y por los adelantos que la humanidad quiera asumir. Lo que sí tengo claro es que a las mujeres nos espera una buena época de ilusión y estimulo, época de pioneras, paritaria a la de los hombres, que cometeremos errores y fallos, como ahora muchos hombres los tienen, y que con buena voluntad los corrigen y corregirán las féminas. Estoy segura de que cuando llegue, pronto, esa paridad entre los sexos en puestos directivos el CSIC se enriquecerá con ese 50 por ciento de mujeres científicas, que entrarán con fuerza a regir los destinos del Consejo y, por lo tanto, de la ciencia, y se notará, como ya se nota ahora, aunque solo sea por el trabajo del día a día llevado a cabo por todos con los proyectos de investigación.

\section{Ana Vieitez. Instituto de Investigaciones Agrobiológicas de Galicia (Santiago de Compostela)}

\section{Experiencia personal}

Mi experiencia personal dentro del CSIC, como mujer, ha sido y es todavía extraordinariamente positiva. Mi único interés ha sido, casi en exclusiva, la carrera investigadora, y en este aspecto no he tenido el más mínimo escollo por el hecho de ser mujer. En mi promoción a Profesor de Investigación no me he sentido desplazada por razón a mi sexo. He dirigido y dirijo todavía un grupo de investigación en el que conviven hombres y mujeres, en donde existen los problemas típicos de todo grupo de trabajo pero no están relacionados con la condición del sexo. El hecho de que haya sido investigadora principal en diferentes proyectos de ámbito nacional y europeo, así como que en el momento actual sea editora asociada en tres revistas internacionales del Science Citation Index indica que mi labor, como investigadora, no se ha visto constreñida por el hecho de ser mujer.

Por una decisión personal, nunca me han interesado los cargos directivos y, por tanto, no tengo ninguna experiencia negativa en este aspecto de enfrentamiento con el sexo opuesto.

El caso concreto de mi Instituto es un poco atípico ya que el predominio de mujeres es bastante notable: de 15 investigadores de plantilla solo $4(27 \%)$ son hombres y en mi grupo de trabajo de 16 personas (incluidos becarios y personal conexo) sólo $6(37.5 \%)$ son hombres. No es de extrañar que en los últimos 20 años sólo hayamos tenido mujeres como directoras de Centro y que en la actualidad la coordinadora institucional del CSIC en Galicia sea también mujer (investigadora de mi Centro). 
Mujer, ciencia y sociedad: las ciencias agrarias

En el ámbito de toda el área los datos no son tan espectaculares pues, según los datos de que dispongo, sólo el 40 por ciento del personal científico son mujeres, pero pienso que deben de ser de los porcentajes de mujeres más altos de las áreas del CSIC. El hecho puede deberse a que, en principio, se nutre de las licenciaturas de Biología, Farmacia y Química donde la presencia de la mujer es muy importante. Lamentablemente, este porcentaje femenino no tiene su reflejo en los cargos directivos del CSIC, en los que, por ejemplo, la Comisión de Área de Ciencias Agrarias está compuesta sólo por hombres y solamente tres mujeres dirigen centros de los doce que tiene Agrarias. Por tanto, parece existir una diferencia notable cuando se trata de un aspecto competitivo (entrada y desarrollo de la carrera investigadora) o un aspecto de designación directa, en cuyo caso la mujer parece perder protagonismo. Mi experiencia en tribunales del CSIC me indica que tanto el acceso como la promoción no están relacionadas con el sexo y sólo cuando se trata de designar cargos claramente pierde la mujer.

\section{Problemas que genera la familia}

Personalmente pienso que éste es el gran problema que tiene la mujer investigadora. Todavía se sigue pensando en este país que la mujer ha de ser la responsable de la casa en general y de los hijos en particular. La legislación se ha ido abriendo más pero, en mis años con hijos pequeños, las facilidades sociales eran mínimas y la mujer era la única responsable del cuidado de los hijos. Como siempre he tenido el máximo interés en desarrollar mi carrera científica, compaginar ambas facetas nos ha supuesto un notable sacrificio económico en términos de sueldos a personas al cargo de los hijos, guarderías no subvencionadas, etc. Hoy día creo que la situación no ha cambiado mucho: cuando algún niño de alguna compañera está enfermo, suele ser la madre quien se "sacrifica» y no el padre, aunque éste tenga una profesión más liberal que la mujer. Si la responsabilidad fuese realmente compartida por los dos cónyuges, el rendimiento de la mujer en la carrera científica podría ser mejor.

\section{Aportaciones de las mujeres científicas}

Entiendo que la mujer científica debe aportar, al igual que el hombre, la mejor ciencia posible. No veo que por el hecho de ser mujer haya que 
aportar algún aspecto extra que nos diferencie de los hombres. Distinto sería en los casos de cargos directivos, donde la mujer podría aportar cierta dosis de calma y tranquilidad que, a veces, no parecen mostrar los hombres. El hombre puede ser más pragmático y la mujer más detallista aunque no estoy muy segura de estas consideraciones, por ser también tópicos de la sociedad. En definitiva, pienso que esto puede estar más relacionado con la personalidad de cada uno que con el hecho de ser mujer $\mathrm{u}$ hombre.

\section{Perspectivas de futuro}

La consolidación de la mujer en el CSIC va a estar relacionada con las oportunidades que se ofrezcan. Cuanto mayor sean esas oportunidades, mayor va a ser la competencia y, por tanto, habrá más dificultades de acceso. Hace ya unos años perdimos en mi grupo de trabajo a cuatro becarios hombres en dos años (algunos con la tesis en fase de redacción) porque las expectativas de consolidación de puestos de trabajo en el CSIC eran muy escasas y «no podían» esperar tanto tiempo. La mujer tiene más capacidad de aguante, estando soltera o casada, y prefiere esperar su oportunidad. Si la oferta de plazas es alta, tanto los hombres como las mujeres esperarían menos y, en consecuencia, la competitividad sería mayor. Por tanto, creo que no puede predecirse el futuro de la mujer en el CSIC porque está sujeto a aspectos coyunturales.

\section{Anunciación Abadía. Estación Experimental de Aula Dei (Zaragoza)}

Empecé a trabajar como becaria honoraria en el Departamento de Nutrición Vegetal de la Estación Experimental de Aula Dei en Zaragoza en el año 1980 y en este centro desarrollé posteriormente mi Tesis Doctoral, presentada en 1985. Después de una estancia postdoctoral de dos años en el extranjero, logré plaza como Científico Titular en 1987. No obstante, mi toma de contacto con la carrera investigadora es incluso anterior a mi época de becaria, ya que la investigación ha estado desde siempre presente en mi ámbito familiar. Quizá por ello veo el trabajo de un investigador como un trabajo normal que puede ser realizado con una amalgama de paciencia, interés, imaginación, curiosidad e ilusión por lo que estás haciendo. 
Mujer, ciencia y sociedad: las ciencias agrarias

\section{Participación de la mujer en investigación}

La evolución de la situación de la mujer en la investigación creo que ha seguido el ritmo de la incorporación de las mujeres al mercado laboral. Desde mi experiencia personal, no creo que haya habido más trabas para que la mujer accediese a la investigación que las que ella misma haya querido imponerse, en general por razones familiares. Por ejemplo, en nuestro Centro, en 1987, entre el personal Científico de plantilla sólo había tres mujeres (una Investigadora y dos Colaboradoras Científicas, lo que suponía menos de un $20 \%$ del total de investigadores). En este momento somos diez (una Investigadora y nueve Científicas Titulares) de un total de 27 investigadores en plantilla, es decir, casi se ha duplicado el porcentaje en los últimos quince años. Además, la mayoría de las personas solicitantes de becas predoctorales que en la actualidad acuden a nuestro Centro en cualquiera de los cuatro Departamentos existentes son mujeres y creo que es una pauta bastante general. Así, en la última convocatoria de becas FPI y dentro de mi área (AGL-Agricultura) un $62 \%$ de las becas fueron concedidas a mujeres. Sin duda, esta situación dará lugar a un considerable aumento en la participación femenina, al menos en este área del CSIC, durante los próximos años.

\section{Influencia del entorno social y familiar}

Este previsible aumento de la participación femenina puede verse frenado por algunos obstáculos y casi todos derivan del papel familiar representado por la mujer como única persona capaz de cuidar de la casa y la familia. El hombre y la mujer se han repartido tradicionalmente tareas en dos extremos opuestos, el hombre en el trabajo fuera de casa y la mujer en las tareas del hogar, aunque también ayudara fuera de la casa. Poco a poco se van aproximando las posiciones, pero todavía hay un amplio abanico de situaciones intermedias y cada caso es un caso particular. En todos los casos es una tarea de toda la familia, y no sólo de la mujer, conseguir que todos sus miembros se puedan desarrollar de acuerdo con sus preferencias.

Es evidente que en el caso de una mujer trabajadora fuera de casa, en éste y en cualquier otro trabajo, la existencia de personas que dependan de una puede suponer una desventaja en el terreno profesional. La libertad para desplazarse, sobre todo en el caso de estancias largas en el extranjero, puede verse muy limitada. Además, el trabajo en investigación requiere muchas veces una total dedicación en tiempo y en esfuerzo, lo 
que es difícil de compaginar con una vida familiar equilibrada. En contraposición a estos problemas, la tarea investigadora realizada en el CSIC ofrece una libertad que no ofrecen otro tipo de trabajos con horarios demasiado rígidos y encorsetados. Y lo que es más importante, la investigación ofrece la posibilidad de ganarte la vida haciendo algo que te gusta, lo que indudablemente va a enriquecer tu vida y las vidas de los que la comparten.

El hecho de que una vez finalizada la tesis doctoral el camino a recorrer por el investigador sea incierto, con un número siempre indeterminado de años en estancias en el extranjero y en contratos más o menos precarios, condiciona la carrera investigadora de hombres y mujeres. Esta preparación, de duración desconocida a priori, incita a algunas personas a buscar opciones laborales más seguras, y es posible que la mujer sea más proclive a decantarse por estas otras opciones, sobre todo si tiene un entorno familiar que limita sus actividades.

\section{¿Es diferente nuestra percepción de la investigación?}

En la manera de vivir de cualquier persona hay un sello propio que, en muchas ocasiones, está marcado por el sexo. Así, creo que la percepción de la mujer ante el mundo del trabajo es, en general, distinta a la del hombre. En el mundo de la investigación ocurre lo mismo, sin que esto quiera decir ni mejor ni peor. Simplemente diferente. Tanto las prioridades como los intereses de la mujer creo que son muy variados y que hay opciones a las que las mujeres no renunciarían, y en esto tengo que dar una opinión muy personal. Quizás este comportamiento influya en la escasa presencia femenina en puestos de responsabilidad si bien es cierto que, al ser puestos generalmente de designación directa, hay otros factores que pueden explicar este hecho. También en el trabajo cotidiano considero que la visión de la mujer y del hombre son distintas : la mujer analiza más pormenorizadamente y se detiene en todos los detalles, mientras que el hombre tiene una visión más global de los temas. Por ello, pienso que son dos maneras de plantear las cosas que se pueden complementar muy bien y pueden mejorar notablemente los resultados alcanzados en las tareas investigadoras.

\section{A modo de conclusión}

Lo que he pretendido con estas escasas líneas es ofrecer mi punto de vista sobre el pasado, el presente y, espero, el prometedor futuro de la 
Mujer, ciencia y sociedad: las ciencias agrarias

mujer en el mundo de la investigación científica. Es un mundo en el que hombres y mujeres tienen que recorrer un camino atractivo y difícil, algunas veces más complicado para ellas que para ellos, pero siempre con la seguridad de estar haciendo algo que han escogido con libertad y que les motiva a seguir trabajando con ilusión. 